CLINICAL STUDY

\title{
Low sex hormone-binding globulin as a predictive marker for insulin resistance in women with hyperandrogenic syndrome
}

Natia Kajaia, Helge Binder, Ralf Dittrich, Patricia G Oppelt, Bianca Flor, Susanne Cupisti, Matthias W Beckmann and Andreas Mueller

Department of Obstetrics and Gynecology, Erlangen University Hospital, Universitaetsstrasse 21-23, D-91054 Erlangen, Germany

(Correspondence should be addressed to A Mueller; Email: andreas.mueller@gyn.imed.uni-erlangen.de)

\begin{abstract}
Background: The aim of the present study is to assess insulin resistance (IR) in women with hyperandrogenic syndrome, which was suggested to replace the term polycystic ovary syndrome by the Androgen Excess Society, and to evaluate whether sex hormone-binding globulin (SHBG) can be used as a predictive marker of IR in hyperandrogenic women.

Methods: Clinical, metabolic, and endocrine parameters were measured, and an oral glucose tolerance test was carried out. The women were classified as IR group or non-IR group, in accordance with defined cutoff points for the homeostatic model assessment of IR (HOMA-IR) at $\geq 2.5$, the quantitative insulin sensitivity check index at $\leq 0.33$, and the Matsuda insulin sensitivity index (ISI) at $\leq 5$.

Results: The women classified as having IR had a significantly higher body mass index (BMI) and free androgen index (FAI) and showed significantly lower SHBG and high-density lipoprotein (HDL) levels, regardless of the indices used. However, with the Matsuda ISI, generally more women were diagnosed as having IR, and this group had significantly higher total testosterone and triglyceride values, as well as a higher incidence of hirsutism.

Conclusions: Women who were classified as being insulin resistant using insulin sensitivity indices showed significantly higher BMI and FAI values and lower SHBG and HDL levels. However, the Matsuda ISI may be more favorable for identifying IR in hyperandrogenic women. SHBG may serve as a predictive marker of IR in these women, particularly in those who are obese.
\end{abstract}

European Journal of Endocrinology 157 499-507

\section{Introduction}

The most common endocrine disorder with the clinical and biochemical features of hyperandrogenemia seen in gynecological practice is polycystic ovary syndrome (PCOS) (1). The syndrome is associated with gynecological, dermatological, and metabolic changes. It is a collection of signs and features in which no single phenomenon is diagnostically relevant on its own (2). The definition of the syndrome has continued to generate significant controversy $(2,3)$.

On the basis of the definition of PCOS, it is possible that completely different phenotypes of the condition exist $(2,3)$. Recently, the Androgen Excess Society suggested that the original National Institutes of Health criteria should be accepted with a few modifications, defining PCOS as an androgen excess syndrome or 'hyperandrogenic syndrome' (2).

Approximately $50-70 \%$ of women with the condition known as PCOS were described as showing hyperinsulinemic insulin resistance (IR), which may play a major pathological role in the development of the syndrome
(4). IR also has a causative role in the development of the metabolic syndrome (MS) $(5,6)$, with severe endocrine and metabolic disturbances leading to several complications later in life, such as cardiovascular diseases (7) and type 2 diabetes (8) and resulting in higher morbidity and mortality, placing a high financial burden on healthcare costs in the 21st century $(9,10)$. A diagnosis of PCOS therefore implies an increased risk of these complications developing later in life.

The earliest diagnosis of IR in women may be possible at the time when hyperandrogenemia-associated symptoms first become manifest. Women with hyperandrogenic syndrome are thus an ideal group for identifying IR at an early stage and preventing its complications later in life $(11,12)$. There are several methods of diagnosing IR. The reference measurement procedure is the hyperinsulinemic euglycemic clamp (13), but this is a complex and time-consuming method of assessing IR (12). Several calculation models using insulin sensitivity indices have therefore been developed for diagnosing IR in clinical practice, and these may also be more useful for epidemiological studies. Despite these 
calculation models, which allow easier and faster assessment, some investigators have advocated the use of sex hormone-binding globulin (SHBG) as a predictive marker of IR in women with hyperandrogenic syndrome (14). However, some groups have only described very small populations of women with PCOS $(15,16)$, or used the Rotterdam criteria to define PCOS (17), while other authors have described the phenomenon additionally in postmenopausal women (18) or in obese children without changes in their androgenic status (19). Recently, cutoff points for insulin sensitivity indices for the simple identification of insulin-resistant patients have been defined (20).

The aims of the present study were to evaluate IR in women with the hyperandrogenic syndrome (2), using different mathematical models and indices for assessing IR, and to evaluate whether SHBG may be able to serve as a predictive marker of IR in hyperandrogenic women.

\section{Materials and methods}

\section{Patients}

During the study period, 214 women were referred to the Division of Gynecological Endocrinology and Reproductive Medicine for the evaluation of possible androgen excess or hyperandrogenism. According to the definition of the Androgen Excess Society, PCOS is above all a disorder of androgen excess in women. As such, with currently available evidence, the diagnosis of PCOS cannot be clearly established without evidence of either clinical or biochemical hyperandrogenism (2), so the inclusion criteria were:

1. Androgen excess and/or

2. Clinical hyperandrogenism (e.g. hirsutism, acne, seborrhea, and/or menstrual dysfunction, e.g. oligomenorrhea or amenorrhea in the absence of other endocrine abnormalities also affecting ovulatory function, such as hyperprolactinemia, functional hypothalamic amenorrhea, or thyroid dysfunction).

Androgen excess was defined by the laboratory as total testosterone (TT) $>2.08 \mathrm{nmol} / \mathrm{l}$ or DHEAS $>6.6 \mu \mathrm{mol} / \mathrm{l}$. Ovulatory and menstrual dysfunction was defined as oligomenorrhea in women with menstrual bleeding intervals longer than 35 days and as amenorrhea in women with absence of any bleeding within the last 6 months $(2,21)$. Polycystic ovarian morphology on ultrasound was not used to enroll the women into the study.

The criteria for exclusion were 21-hydroxylasedeficient nonclassical adrenal hyperplasia (NCAH), hyperandrogenic insulin-resistance acanthosis nigricans (HAIRAN) syndrome, or an androgen-secreting neoplasm (ASN). Women who had been receiving hormonal therapy, including oral contraceptive pills or steroid medications, within 3 months of their initial visit were not included. The study was approved by the local ethics committee. All the patients provided written informed consent and completed a standard medical history questionnaire, with an emphasis on menstrual dates and regularity, hirsutism, acne, gynecological history, history of infertility, medications, and family medical history.

\section{Procedures}

All the women underwent a complete screening panel, including physical examination, weight and height measurement, ultrasound examination of the ovaries, and calculation of the body mass index (BMI). The interval between menstrual periods was assessed. Women with amenorrhea within the previous year were categorized as anovulatory without further testing, and blood was taken for hormonal analysis immediately. In women with menstrual cycles, serum was obtained between days 3 and 5 of their menstrual cycle. Women with cycles of less than 26 days were not included in the study.

\section{Calculation of IR}

All the patients were on an unrestricted diet. An oral glucose tolerance test $(75 \mathrm{~g})$ was carried out, with glucose $(\mathrm{mg} / \mathrm{dl})$ and insulin $(\mu \mathrm{IU} / \mathrm{ml})$ measured at 0,60 , and $120 \mathrm{~min}$. After the glucose and insulin levels had been measured, the following mathematical models were used to assess IR:

The homeostatic model assessment of IR (HOMA-IR) was calculated using the following formula (22):

Fasting glucose $(\mathrm{FG}, \mathrm{mmol} / \mathrm{l}) \times$ fasting insulin $(\mathrm{FI}, \mu \mathrm{U} / \mathrm{ml})$

$$
22.5
$$

The HOMA of $\beta$-cell function (HOMA-B) was calculated using the following formula (22):

$$
\frac{20 \times \mathrm{FI}(\mu \mathrm{U} / \mathrm{ml})}{(\mathrm{FG}(\mathrm{mmol} / \mathrm{l})-3.5)}
$$

The quantitative insulin sensitivity check index (QUICKI) was calculated using the following formula (23):

$$
\frac{1}{(\log (\mathrm{FI})(\mu \mathrm{U} / \mathrm{ml})+\log (\mathrm{FG})(\mathrm{mg} / \mathrm{dl}))}
$$

The insulin sensitivity index (ISI) was calculated using the following formula (24): 
10000

$\sqrt{(\mathrm{FG}(\mathrm{mg} / \mathrm{dl}) \times \mathrm{FI}(\mu \mathrm{U} / \mathrm{ml}) \times \mathrm{G}(\mathrm{mg} / \mathrm{dl}) \times \mathrm{I}(\mu \mathrm{U} / \mathrm{ml}))}$

where $G=$ mean plasma glucose concentration and $I=$ mean insulin concentration during oral glucose tolerance test (OGTT).

Women were classified as being insulin resistant (IR group) or not insulin resistant (non-IR group) in accordance with defined cutoff points for HOMA-IR $\geq 2.5(25,26)$, QUICKI $\leq 0.33(26,27)$, and ISI $\leq 5$ $(20,24)$.

\section{Exclusion of related disorders}

For evaluation of an ASN, the TT cutoff value used was above $7 \mathrm{nmol} / \mathrm{l}$, at which point computed tomography of the adrenal gland is normally carried out at our institution to exclude an ASN. To exclude 21-hydroxylase deficiency in patients with a 17-hydroxyprogesterone (17-HP) level above $6 \mathrm{nmol} / \mathrm{l}, 17-\mathrm{HP}$ levels stimulated by adrenocorticotropic hormone (ACTH) were measured $(28,29)$. Briefly, all tests were started between 0800 and $0900 \mathrm{~h}$ with the patients in the fasting state. A baseline sample was obtained, and afterward $0.25 \mathrm{mg}$ ACTH (tetracosactide/Synacthen, Novartis) was administered i.v. over $60 \mathrm{~s}$ and blood was sampled $60 \mathrm{~min}$ later. Both the baseline and 60-min samples were assayed for 17-HP levels. If the stimulated 17 -HP level was greater than $30 \mathrm{nmol} / \mathrm{l}$, the woman was considered to have 21-hydroxylase deficiency NCAH.

\section{Biochemical measurements}

All the assays were carried out in our routine diagnostic endocrinology laboratory, using established commercial assays routinely monitored by participation in external quality control programs. All samples were obtained between 0800 and $1000 \mathrm{~h} \mathrm{(30).}$

TT, DHEAS, and SHBG were measured with chemiluminescent enzyme immunoassays (Immulite 2000, Diagnostic Products Corporation, Los Angeles, CA, USA). The calibration range of the TT assay was $0.7-55 \mathrm{nmol} / \mathrm{l}$, with an analytical sensitivity of $0.5 \mathrm{nmol} / \mathrm{l}$. The cross-reaction with 5 - $\alpha$-dihydrotestosterone was $2 \%$. The calibration range of the DHEAS assay was $0.41-27 \mu \mathrm{mol} / \mathrm{l}$, with an analytical sensitivity of $0.08 \mu \mathrm{mol} / \mathrm{l}$. No cross-reactivity with other compounds was known. The calibration range of the SHBG assay was up to $180 \mathrm{nmol} / \mathrm{l}$, with an analytical sensitivity of $0.02 \mathrm{nmol} / \mathrm{l}$. The inter-assay and intraassay coefficients of variation (CV) were always below $11 \%$ at mid-range concentrations. No cross-reactivity with other compounds is known.

Estradiol was measured using a solid-phase competitive chemiluminescent enzyme immunoassay (Immulite 2000, Diagnostic Products Corporation). The calibration range of the assay was $73-7342 \mathrm{pmol} / \mathrm{l}$, with an analytical sensitivity of $55 \mathrm{pmol} / \mathrm{l}$. The intra-assay CV values were $9.9,7.8$, and $4.3 \%$ at the levels of 327,660 , and $1692 \mathrm{pmol} / \mathrm{l}$ respectively. The corresponding interassay CV values were 16,11 , and $6.7 \%$. The crossreactivity with $17 \beta$-estradiol valerate was $1.14 \%$.

Prolactin (PRL) was measured using an immunometric assay (Immulite 2000, Diagnostic Products Corporation). The calibration range of the assay was up to $3180 \mathrm{mIU} / \mathrm{l}$, with an analytical sensitivity of $3.4 \mathrm{mIU} / \mathrm{l}$. The intraassay $\mathrm{CV}$ values were $2.8,3.6$, and $2.3 \%$ at the levels of 186.6, 402.6, and $466.6 \mathrm{mIU} / \mathrm{l}$. The corresponding interassay $\mathrm{CV}$ values were $8.2,7.4$, and $5.9 \%$. No crossreactivity with other compounds is known.

Luteinizing hormone (LH) was measured with an immunometric assay (Immulite 2000, Diagnostic Products Corporation). The calibration range of the assay was up to $200 \mathrm{mIU} / \mathrm{ml}$, with an analytical sensitivity of $0.05 \mathrm{mIU} / \mathrm{ml}$. The intra-assay $\mathrm{CV}$ values were $3.04,3.71$, and $3.6 \%$ at the levels of $1.04,1.89$, and $8.7 \mathrm{mIU} / \mathrm{ml}$. The corresponding inter-assay $\mathrm{CV}$ values were $6.6,6.2$, and $6.7 \%$. The cross-reactivity with human chorionic gonadotropin was $0.20 \%$.

Follicle-stimulating hormone (FSH) was measured with an immunometric assay (Immulite 2000, Diagnostic Products Corporation). The calibration range of the assay was up to $170 \mathrm{mIU} / \mathrm{ml}$, with an analytical sensitivity of $0.1 \mathrm{mIU} / \mathrm{ml}$. The intra-assay $\mathrm{CV}$ values were $2.5,2.9$, and $2.1 \%$ at the levels of $4,9.1$, and $40 \mathrm{mIU} / \mathrm{ml}$. The corresponding inter-assay $\mathrm{CV}$ values were $6.3,5.5$, and $4.3 \%$. The cross-reactivity with thyroid-stimulating hormone was $0.01 \%$.

Plasma insulin was determined using a solid-phase two-site chemiluminescent immunometric assay (Immulite 2000, Diagnostic Products Corporation). The calibration range of the assay was up to $300 \mu \mathrm{IU} / \mathrm{ml}$, with an analytical sensitivity of $2 \mu \mathrm{IU} / \mathrm{ml}$. The intra-assay CV values were $5.5,4.0,3.3,3.9,3.8$, and $3.7 \%$ at the levels of $7.67,12.5,17.2,26.4,100$, and $291 \mu \mathrm{IU} / \mathrm{ml}$. The corresponding inter-assay CV values were $7.3,4.9,4.1,5.0,4.2$, and $5.3 \%$. The crossreactivity with proinsulin was $8 \%$. The plasma concentration was measured with the glucose oxidase method, using an automatic biochemical analyzer (Immulite 2000, Diagnostic Products Corporation). Total cholesterol (TC), low-density lipoprotein (LDL), high-density lipoprotein (HDL), and triglycerides (TG) were regularly measured after an overnight fasting period of $12 \mathrm{~h}$, using routine clinical chemistry methods, and documented.

\section{Calculation of the free androgen index (FAI)}

The FAI was calculated as the quotient $100 \times$ TT/SHBG (31).

\section{Statistical analysis}

All the data are presented as means \pm s.D. unless otherwise stated. We employed nonparametric statistical 
tests, which are based on ranks of observations and require no assumptions about the underlying distribution of data. All hypothesis tests were two sided and conducted at the 0.05 significance level. Two-sample Wilcoxon tests (i.e., Wilcoxon rank-sum tests) were used to compare parameters between women in the IR group and women in the non-IR group. We explored the association of SHBG with HOMA-IR, QUICKI, and Matsuda ISI using simple Spearman's rank-order correlation procedures; the resulting estimates of rho are reported as $r_{\mathrm{s}}$ values. In addition, it was assessed whether clinical manifestations of hyperandrogenemia (hirsutism, acne, and ultrasound evidence of polycystic ovaries) were more frequently found in the IR group or non-IR group using the $\chi^{2}$-test of independence. All the statistical analyses were carried out using the Statistical Package for the Social Sciences (SPSS, version 13.0 for Windows; SPSS Inc., Chicago, IL, USA).

\section{Results}

A total of 214 women with clinical or biochemical signs of hyperandrogenemia presented for the evaluation of possible androgen excess or hyperandrogenism at the Division of Gynecological Endocrinology and Reproductive Medicine in Erlangen University Hospital between January 2005 and December 2006. Nineteen women were not included, as they had been receiving hormonal treatment within 3 months of their initial visit. Eight women had hyperprolactinemic oligo-/anovulation, and two women showed evidence of 21-OHdeficient NCAH. All the women included in the study had at least one raised androgen level. No ovarian tumors were identified using ultrasonography in any of the women, and none of them had HAIRAN syndrome. The study population thus consisted of 185 women with a hyperandrogenic syndrome.

\section{Comparison of women in the IR group and non-IR group}

The anthropometric and metabolic parameters, steroid hormone levels, SHBG levels, and lipid parameters in the IR group and non-IR group using the different mathematical indexes (HOMA, QUICKI, and Matsuda ISI) are shown in Table 1.

\section{HOMA-IR}

A total of 71 women were classified as having IR in accordance with a HOMA-IR value of $\geq 2.5$. There were no significant differences between the groups with regard to age or levels of LH, FSH, PRL, estradiol (E), TT, DHEAS, TC, TG, or LDL. Women classified as having IR had significantly higher values for BMI, FG, G-1h, G-2h, FI, I-1h, I-2h, and HOMA-B, and significantly lower values for the glucose infusion rate (GIR), QUICKI, and
Matsuda ISI. Women classified as having IR had significantly lower SHBG and HDL values and higher FAI values. No differences were found between the women in the two groups with regard to possible clinical manifestations of hyperandrogenemia.

\section{QUICKI}

A total of 74 women were classified as having IR in accordance with a QUICKI value of $\leq 0.33$. There were no significant differences in age, LH, FSH, PRL, E, TT, DHEAS, TC, TG, or LDL between the two groups. Women classified as having IR had significantly higher values for BMI, FG, G-1h, G-2h, FI, I-1h, I-2h, HOMA IR, and HOMA B, and significantly lower values for GIR and Matsuda ISI. Women classified as having IR had significantly lower SHBG and HDL and higher FAI values. No differences were found between the women in the two groups with regard to possible clinical manifestations of hyperandrogenemia.

\section{ISI}

A total of 91 women were classified as having IR in accordance with an ISI value of $\leq 5$. There were no significant differences in age, LH, FSH, PRL, E, DHEAS, TC, or LDL between the two groups. Women classified as having IR had significantly higher values for BMI, FG, G-1h, G-2h, FI, I-1h, I-2h, HOMA IR, and HOMA B, and significantly lower values for GIR and QUICKI. Women classified as having IR had significantly lower SHBG and HDL and higher FAI, TT, and TG values. Women in the IR group had hirsutism significantly more often.

\section{Spearman's rank-order correlations}

There was a negative correlation between SHBG and HOMA-IR, with estimated $\rho\left(r_{\mathrm{s}}\right)$ values of -0.491 for all the women (Fig. 1), -0.324 for women in the IR group, and -0.309 for women in the non-IR group. There was a positive correlation between SHBG and QUICKI, with estimated rho $\left(r_{\mathrm{s}}\right)$ values of 0.423 for all the women (Fig. 2), 0.352 for women in the IR group, and 0.310 for women in the non-IR group. There was a positive correlation between SHBG and Matsuda ISI, with estimated rho $\left(r_{\mathrm{s}}\right)$ values of 0.539 for all the women (Fig. 3), 0.413 for women in the IR group, and 0.285 for women in the non-IR group (Table 2).

\section{Discussion}

The study was conducted in order to assess IR among women with the hyperandrogenic syndrome (2), using different mathematical models and indices for assessing IR, and to evaluate whether SHBG might be able to serve as a predictive marker of IR in hyperandrogenic women. In this study, hyperandrogenic women 
Table 1 Two-sample Wilcoxon comparisons of the different mathematical models for assessment of insulin resistance in hyperandrogenic women.

\begin{tabular}{|c|c|c|c|c|c|c|}
\hline & HOMA $\geq 2.5$ & HOMA $<2.5$ & QUICKI $\leq \mathbf{0 . 3 3}$ & QUICKI >0.33 & ISI Matsuda $\leq 5$ & ISI Matsuda $>5$ \\
\hline Patients $(n=185)$ & 71 & 114 & 74 & 111 & 91 & 94 \\
\hline & $28.09 \pm 6.71$ & $28.16 \pm 6.93$ & $28.07 \pm 6.66$ & $28.16 \pm 6.97$ & $28.01 \pm 6.75$ & $28.22 \pm 6.94$ \\
\hline $\mathrm{BMI}\left(\mathrm{kg} / \mathrm{m}^{2}\right)$ & $34.09 \pm 7.60$ & $25.13 \pm 5.81^{\ddagger}$ & $33.91 \pm 7.64$ & $25.09 \pm 5.81^{\ddagger}$ & $32.82 \pm 8.03$ & $24.55 \pm 5.14^{\ddagger}$ \\
\hline $\mathrm{FG}(\mathrm{mg} / \mathrm{dl})$ & $91.06 \pm 8.75$ & $82.22 \pm 7.11^{\ddagger}$ & $90.92 \pm 8.71$ & $92.18 \pm 7.13^{\ddagger}$ & $89.69 \pm 8.79$ & $81.77 \pm 7.08^{\ddagger}$ \\
\hline $\mathrm{G}-1 \mathrm{~h}(\mathrm{mg} / \mathrm{dl})$ & $152.69 \pm 40.66$ & $116.10 \pm 40.23^{\ddagger}$ & $151.25 \pm 41.89$ & $116.40 \pm 39.96^{\ddagger}$ & $154.58 \pm 38.53$ & $107.06 \pm 35.85^{\ddagger}$ \\
\hline G-2h (mg/dl) & $122.17 \pm 36.03$ & $95.07 \pm 26.20^{\ddagger}$ & $121.48 \pm 35.48$ & $95.05 \pm 26.26^{\ddagger}$ & $121.95 \pm 35.64$ & $89.92 \pm 20.70^{\ddagger}$ \\
\hline $\mathrm{FI}(\mu \mathrm{U} / \mathrm{ml})$ & $19.11 \pm 10.24$ & $6.08 \pm 2.54^{\ddagger}$ & $18.41 \pm 10.98$ & $5.99 \pm 2.46^{\ddagger}$ & $16.87 \pm 10.12$ & $5.63 \pm 2.46^{\ddagger}$ \\
\hline $\mathrm{l}-1 \mathrm{~h}(\mu \mathrm{U} / \mathrm{ml})$ & $153.25 \pm 99.54$ & $67.46 \pm 50.73^{\ddagger}$ & $152.04 \pm 98.79$ & $66.74 \pm 50.44^{\ddagger}$ & $152.72 \pm 93.76$ & $50.98 \pm 22.46^{\ddagger}$ \\
\hline $\mathrm{I}-2 \mathrm{~h}(\mu \mathrm{U} / \mathrm{ml})$ & $134.62 \pm 105.93$ & $50.81 \pm 48.82^{\ddagger}$ & $132.93 \pm 105.07$ & $50.44 \pm 42.04^{\ddagger}$ & $132.14 \pm 101.12$ & $36.58 \pm 18.49^{\ddagger}$ \\
\hline GIR & \pm 0.99 & $8.51^{\ddagger}$ & $5.74 \pm 2.14$ & $16.65 \pm 8.50^{\ddagger}$ & $6.70 \pm 2.97$ & $17.67 \pm 8.78^{\ddagger}$ \\
\hline HOMA-IR & $4.31 \pm 2.49$ & $1.24 \pm 0.54^{\ddagger}$ & $4.26 \pm 2.48$ & $1.22 \pm 0.53^{\ddagger}$ & $3.77 \pm 2.45$ & $1.14 \pm 0.52^{\ddagger}$ \\
\hline HOMA-B & $273.89 \pm 178.44$ & $130.62 \pm 89.23^{\ddagger}$ & $271.65 \pm 176.64$ & $129.56 \pm 89.42^{\ddagger}$ & $249.27 \pm 168.48$ & $125.61 \pm 92.21^{\ddagger}$ \\
\hline & & & & & & $5 \pm 4.74^{\ddagger}$ \\
\hline ICKI & & & & $0.38 \pm$ & & $03^{\ddagger}$ \\
\hline LH (IU/I) & $7.71 \pm 5.71$ & 8.9 & 7.5 & 9.0 & 7.6 & 9.28 \\
\hline FSH (IU/I) & $6.25 \pm 2.93$ & $8.29 \pm$ & 6.2 & \pm 11 & 6.2 & 12.59 \\
\hline PRL (ng/ml) & $14.13 \pm$ & $10 . c$ & & & & \\
\hline & 9.79 & 5.26 & 3.82 & 143 & 8.88 & 7.17 \\
\hline $\mathrm{ol} / \mathrm{l})$ & & & & & & $14^{\dagger}$ \\
\hline DHEAS (pmol/l) & $6.60 \pm 4.10$ & $7.45 \pm 4.17$ & $6.62 \pm 4.06$ & $7.46 \pm 4.20$ & $7.07 \pm 3.95$ & $7.17 \pm 4.35$ \\
\hline SHBG $(\mathrm{nmol} / \mathrm{l})$ & $28.35 \pm 15.42$ & $46.14 \pm 22.24^{\ddagger}$ & $28.92 \pm 15.94$ & $46.09 \pm 22.28^{\ddagger}$ & $29.51 \pm 15.57$ & $48.55 \pm 22.61^{\ddagger}$ \\
\hline $\mathrm{FAl}$ & 12.83 & $6.78 \pm 5.04^{\ddagger}$ & $12.65 \pm 10.34$ & $6.80 \pm 5.09^{\ddagger}$ & $12.16 \pm 9.58$ & $6.22 \pm 4.93^{\ddagger}$ \\
\hline $\mathrm{TC}(\mathrm{mg} / \mathrm{dl})$ & $202.06 \pm 38.83$ & $199.48 \pm 27.39$ & $202.06 \pm 38.84$ & $199.48 \pm 27$ & $204.97 \pm 35.76$ & 192.80 \\
\hline $\mathrm{TG}(\mathrm{mg} / \mathrm{dl})$ & $168.46 \pm 114.64$ & $142.42 \pm 66.37$ & 14.64 & 142.43 & 104.65 & 121 \\
\hline & $43.96 \pm 12.24$ & $52.95 \pm 12.35^{\dagger}$ & $43.97 \pm 12.24$ & $52.96 \pm 12.35^{\dagger}$ & $45.20 \pm 11.70$ & $53.38 \pm 13.71^{\dagger}$ \\
\hline LDL (mg/dl) & $135.72 \pm 34.65$ & $122.08 \pm 21.92$ & $135.72 \pm 34.66$ & $122.08 \pm 21.92$ & $135.43 \pm 32.20$ & $118.94 \pm 22.25$ \\
\hline \multicolumn{7}{|c|}{ Clinical manifestations of hyperandrogenism } \\
\hline & $n=25(35.2 \%)$ & $n=58(50.8 \%)$ & $n=24(32.4 \%)$ & $n=56(50.4 \%)$ & $n=41(45.1 \%)$ & $n=42(44.7 \%)$ \\
\hline Hirsutism & $n=49(69.1 \%)$ & $n=54(47.3 \%)$ & & $n=50(45.0 \%)$ & $n=67(73.6 \%)$ & $n=36(38.3 \%)^{\dagger}$ \\
\hline PCO & $n=38(53.5 \%)$ & $n=59(51.2 \%)$ & $n=39(52.7 \%)$ & $n=57(51.3 \%)$ & $n=56(61.5 \%)$ & $n=41(43.6 \%)$ \\
\hline
\end{tabular}

Data are shown as means \pm s.D. ${ }^{\star} P<0.05 ;{ }^{\dagger} P<0.01 ;{ }^{\ddagger} P<0.0001$, HOMA IR $\geq 2.5$ versus HOMA $<2.5$; QUICKI $\leq 0.33$ versus QUICKI $>0.33$; ISI Matsuda $\leq 5$ versus ISI Matsuda $>5$. BMI, body mass index; DHEAS, dehydroepiandrosterone; E, estradiol; $\mathrm{FAl}$, free androgen index; $\mathrm{FG}$, fasting glucose; $\mathrm{FI}$, fasting insulin; FSH, follicle-stimulating hormone; G, glucose; GIR, glucose infusion rate; HDL, high-density lipoprotein; HOMA-B, homeostatic model assessment of $\beta$ cell function; HOMA-IR, homeostatic model assessment of insulin resistance; I, insulin; ISI, insulin sensitivity index; LDL, low-density lipoprotein; LH, luteinizing hormone; PCO, polycystic ovaries; PRL, prolactin; QUICKI, quantitative insulin sensitivity check index; SHBG, sex hormone-binding globulin; TC, total cholesterol; TG, triglycerides; TT, total testosterone.

who were identified as having IR were found to have significantly increased BMI levels, and fasting and stimulated glucose and insulin levels, while SHBG and HDL were significantly decreased, regardless of the insulin sensitivity indices used. However, the Matsuda ISI method identified more women as being insulin resistant, and hirsutism was more evident as a major

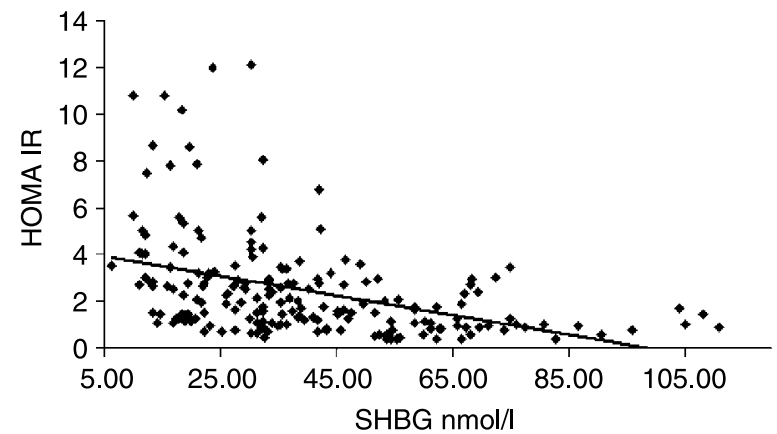

Figure 1 The relationship between sex hormone-binding globulin (SHBG) and the homeostatis model assessment of insulin resistance (HOMA-IR) in hyperandrogenic women $(n=185)$. clinical symptom of androgen excess. In general, there were few or no differences between the two groups with regard to acne, hirsutism, and the ultrasound appearance of the ovaries.

The urgent need for a simple method of measuring IR has led to the development of a large number of insulin sensitivity indices, which have been reviewed elsewhere

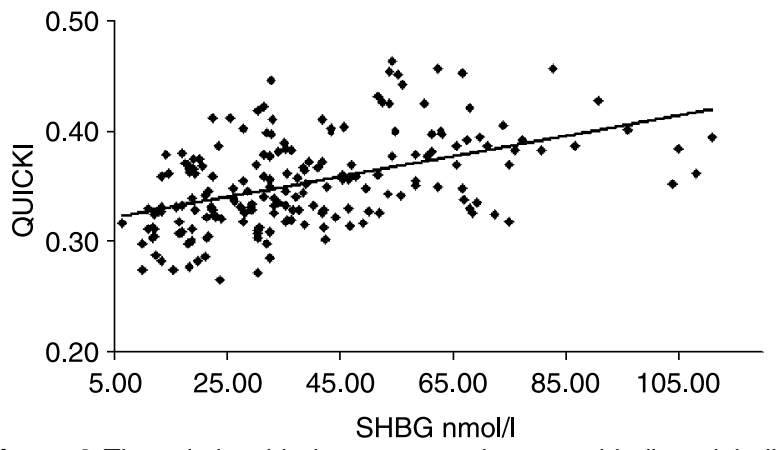

Figure 2 The relationship between sex hormone-binding globulin (SHBG) and the quantitative insulin sensitivity check index (QUICKI) in hyperandrogenic women $(n=185)$. 


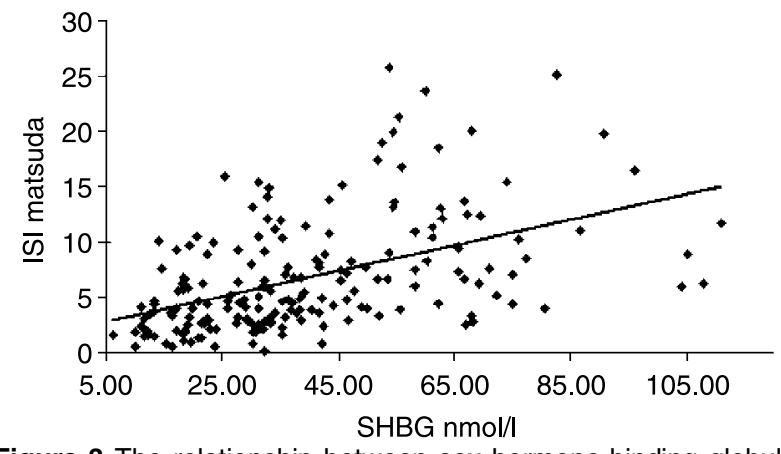

Figure 3 The relationship between sex hormone-binding globulin (SHBG) and the insulin sensitivity index (ISI Matsuda) in hyperandrogenic women $(n=185)$.

(24, 32-34). Recently, reference values have also been established for the Matsuda ISI to distinguish between insulin-resistant and insulin-sensitive patients (20). However, it may be difficult to use the cutoff points for the selected indices, and it should be noted that these indices are not absolutely strict for the diagnosis of impaired carbohydrate metabolism, although they may help identify women who are at the highest risk of developing diabetes (20). Diagnosing IR in women with hyperandrogenic syndrome or androgen excess may help identify endocrine and metabolic risk factors and reveal individuals suitable for targeted treatment with insulin sensitizers, to improve therapeutic approaches and prevent complications later in life $(11,12)$.

SHBG is the main serum transporter of testosterone, while insulin is one of the main modulators of serum SHBG levels (35). The increased concentrations of insulin and androgens suppress SHBG and insulin-like growth factor-binding protein production in the liver (36). This process is enhanced in patients with raised BMI levels (37). A reduced SHBG level is thus not only a possible marker for hyperandrogenism, but has also been postulated as a predictive marker of IR $(38,39)$. Some studies have also suggested using SHBG as a marker of IR in women with PCOS, but these only included small groups of patients $(14,15)$; another study reported no advantage in using SHBG as a predictive marker of IR in 21 women with PCOS, in comparison with

Table 2 Spearman's rank correlations showing estimates of rho.

\begin{tabular}{llll}
\hline SHBG versus & \multicolumn{1}{c}{ All women } & \multicolumn{1}{c}{ IR group } & Non-IR group \\
\hline HOMA-IR & $-0.491^{\ddagger}$ & $-0.324^{*}$ & $-0.309^{\star}$ \\
& $(n=185)$ & $(n=71)$ & $(n=114)$ \\
QUICKI & $0.496^{\ddagger}$ & $0.352^{*}$ & $0.310^{\star}$ \\
& $(n=185)$ & $(n=74)$ & $(n=111)$ \\
ISI Matsuda & $0.539^{\ddagger}$ & $0.413^{\ddagger}$ & $0.285^{\star}$ \\
& $(n=185)$ & $(n=91)$ & $(n=94)$ \\
\hline
\end{tabular}

${ }^{\star} P<0.01 ;{ }^{\ddagger} P<0.0001$. HOMA-IR, homeostatic model assessment of insulin resistance; IR, insulin resistant; ISI, insulin sensitivity index; QUICKI, quantitative insulin sensitivity check index; SHBG, sex hormone-binding globulin.
17 control individuals (40). Dahan \& Goldstein conclude that there is significant variability and overlap in SHBG levels among lean insulin-sensitive control individuals and patients with IR, and despite the statistically significant correlation coefficient, the relationship between the groups was weak. This was explained by the multifactorial regulation of the SHBG concentration and its genetic variability (40). Some studies have emphasized the influence of genetics on circulating SHBG levels. Recently, it was postulated that polymorphisms within the coding sequence and in the regulatory sequence of the SHBG gene were associated with circulating SHBG levels, which may be a possible genetic background of sex hormone activity in humans $(41,42)$. On the other hand, Bendlova et al. reported no significant differences in the genotype distribution between PCOS and controls and no association with plasma SHBG levels (43). Nevertheless, there may be a genetic determination of low SHBG levels contributing to an increase in biologically active androgen levels during fetal, pubertal, and later in life, resulting in different phenotypes of androgen excess (42).

Ducluzeau et al. (44) reported that the GIR is a better marker for assessing IR than SHBG levels in nonobese women with PCOS. The authors also reported that low SHBG levels in PCOS are associated with the BMI, suggesting that certain signals from adipose tissue may regulate the hepatic production of SHBG. It has been reported that SHBG may constitute an index of IR only in the hyperinsulinemic state (45). These results are similar to those of the present study: Spearman' rank correlations for SHBG versus the insulin sensitivity indices used were lowest in the non-IR group and reached a lower level of significance both in comparison with the IR group and also when all women were taken into account. It should be noted that women in the non-IR group had significantly lower BMI values. In general, the results using the HOMA-IR and QUICKI indices were similar. It was only using the Matsuda ISI method that more women were identified as being insulin resistant, and hirsutism as a major clinical symptom of androgen excess was more evident in this group. In addition, women identified as having IR using the Matsuda ISI had significantly higher testosterone levels in comparison with non-IR women.

It has been postulated that insulin sensitivity indices using values obtained during an OGTT may provide greater objectivity in assessing IR than indices calculated from the fasting glucose and insulin values alone $(20,46,47)$. It has also been reported that the Matsuda ISI can best predict the risk of IR, and that it provides the best relative sensitivity and specificity rates in comparison with HOMA-IR $(20,46)$.

SHBG also shares many features with HDL; both are reduced in women with PCOS (48). It has been hypothesized that SHBG affects atherogenesis both directly and indirectly, through the lipoprotein 
metabolism or by affecting the estradiol-testosterone balance (49). In the present study, women identified as having IR had significantly lower HDL concentrations, regardless of the calculation model used. The TG concentration was also higher in women in the IR group, but the difference was only significant with the Matsuda ISI test. Chen et al. conclude that the coexistence of low SHBG and reduced HDL levels is associated with the occurrence of MS, and that it may increase the risk of cardiovascular disease in women with PCOS (17). It was also postulated that in women with type 2 diabetes, androgen excess may contribute to greater burden of coronary heart disease risk factors (50).

Since SHBG binds testosterone, a decrease in SHBG results in a more pronounced increase in circulating free androgens, independently of albumin levels (51, 52 ). In the revised 2003 consensus, it was postulated that calculating free testosterone from measurements of SHBG and TT and calculating the FAI are more sensitive methods of assessing hyperandrogenemia in women with PCOS (53). Whether they meet the Rotterdam criteria or not, hirsute women have significantly increased calculated free and bioavailable testosterone levels in comparison with nonhirsute hyperandrogenic control individuals (54). However, this diagnostic advantage for calculated free and bioavailable testosterone levels was not apparent in PCOS women in comparison with hirsute controls (55). These phenomena support the hypothesis that the definition of PCOS in the revised 2003 Rotterdam criteria identifies a different subset of women who have different endocrine profiles, whether they are hyperandrogenic or not. We recently reported that the BMI is associated with significantly lower SHBG levels in women with hirsutism and PCOS, resulting in significantly increased calculated free testosterone and FAI levels, which were appropriate markers for assessing hyperandrogenemia in these women $(54,56)$.

This study investigated the endocrine and metabolic changes in women with hyperandrogenic syndrome who showed evidence of clinical or biochemical hyperandrogenism. Furthermore, most women met also the Rotterdam criteria for PCOS. Therefore, these findings are not contradictory to the conclusion that PCOS should be understood as a disorder of androgen excess or hyperandrogenism considering the possibility of the different phenotypes.

In summary, despite the controversy over the definition, this study shows that women with hyperandrogenic syndrome who are classified as being insulin resistant using insulin sensitivity indices have significantly higher BMI levels and lower HDL and SHBG levels, resulting in higher FAI values. There appears to be no obvious association with the clinical manifestations of hyperandrogenemia, such as hirsutism, acne, and ultrasound evidence of polycystic ovaries. In addition, the Matsuda ISI may be preferable for identifying IR, and SHBG may serve as predictive marker of IR in all hyperandrogenic women with a better correlation in obese women.

\section{Acknowledgements}

The research for this study was supported by a grant from the Catholic Academic Exchange Service (Katholischer Akademischer Austausch Dienst, KAAD).

\section{References}

1 Ovalle $\mathrm{F}$ \& Azziz R. Insulin resistance, polycystic ovary syndrome, and type 2 diabetes mellitus. Fertility and Sterility 200277 1095-1105.

2 Azziz R, Carmina E, Dewailly D, Diamanti-Kandarakis E, EscobarMorreale HF, Futterweit W, Janssen OE, Legro RS, Norman RJ, Taylor AE \& Witchel SF. Androgen Excess Society. Positions statement: criteria for defining polycystic ovary syndrome as a predominantly hyperandrogenic syndrome: an Androgen Excess Society guideline. Journal of Clinical Endocrinology and Metabolism $2006914237-4245$.

3 Azziz R. Controversy in clinical endocrinology: diagnosis of polycystic ovarian syndrome: the Rotterdam criteria are premature. Journal of Clinical Endocrinology and Metabolism $2006 \mathbf{9 1}$ 781-785.

4 Dunaif A. Insulin resistance and the polycystic ovary syndrome: mechanism and implications for pathogenesis. Endocrine Reviews 199718 774-800.

5 Eckel RH, Grundy SM \& Zimmet PZ. The metabolic syndrome. Lancet $20053651415-1428$.

6 Reaven GM. Banting lecture. Role of insulin resistance in human disease. Diabetes 198837 1595-1607.

7 Isomaa B, Almgren P, Tuomi T, Forsen B, Lahti K, Nissen M, Taskinen MR \& Groop L. Cardiovascular morbidity and mortality associated with the metabolic syndrome. Diabetes Care $20012 \mathbf{2 4}$ 683-689.

8 Meigs JB, Rutter MK, Sullivan LM, Fox CS, D’Agostino RB Sr \& Wilson PW. Impact of insulin resistance on risk of type 2 diabetes and cardiovascular disease in people with metabolic syndrome. Diabetes Care 200730 1219-1225.

9 Economic consequences of diabetes mellitus in the US in 1997. American Diabetes Association. Diabetes Care 199821 296-309.

10 Alberti KG. The costs of non-insulin-dependent diabetes mellitus. Diabetic Medicine 199714 7-9.

11 Ehrmann DA, Barnes RB, Rosenfield RL, Cavaghan MK \& Imperial J. Prevalence of impaired glucose tolerance and diabetes in women with polycystic ovary syndrome. Diabetes Care 199922 141-146.

12 Dahlgren E, Johansson S, Lindstedt G, Knutsson F, Oden A, Janson PO, Mattson LA, Crona N \& Lundberg PA. Women with polycystic ovary syndrome wedge resected in 1956 to 1965: a long-term follow-up focusing on natural history and circulating hormones. Fertility and Sterility 199257 505-513.

13 DeFronzo RA, Tobin JD \& Andres R. Glucose clamp technique: a method for quantifying insulin secretion and resistance. American Journal of Physiology 1979237 E214-E223.

14 Birkeland KI, Hanssen KF, Torjesen PA \& Vaaler S. Level of sex hormone-binding globulin is positively correlated with insulin sensitivity in men with type 2 diabetes. Journal of Clinical Endocrinology and Metabolism 199376 275-278.

15 Jayagopal V, Kilpatrick ES, Jennings PE, Hepburn DA \& Atkin SL. The biological variation of testosterone and sex hormone-binding globulin (SHBG) in polycystic ovarian syndrome: implications for SHBG as a surrogate marker of insulin resistance. Journal of Clinical Endocrinology and Metabolism 200388 1528-1533. 
16 Cibula D, Skrha J, Hill M, Fanta M, Haakova L, Vrbikova J \& Zivny J. Prediction of insulin sensitivity in nonobese women with polycystic ovary syndrome. Journal of Clinical Endocrinology and Metabolism 200287 5821-5825.

17 Chen MJ, Yang WS, Yang JH, Hsiao CK \& Yang YS. Low sex hormone-binding globulin is associated with low high-density lipoprotein cholesterol and metabolic syndrome in women with PCOS. Human Reproduction 200621 1171-2266.

18 Weinberg ME, Manson JE, Buring JE, Cook NR, Seely EW, Ridker PM \& Rexrode KM. Low sex hormone-binding globulin is associated with the metabolic syndrome in postmenopausal women. Metabolism 200655 1473-1480 (erratum in Metabolism 200756 296).

19 Gascon F, Valle M, Martos R, Ruz FJ, Rios R, Montilla P \& Canete R. Sex hormone-binding globulin as a marker for hyperinsulinemia and/or insulin resistance in obese children. European Journal Endocrinology 2000143 85-89.

20 Radikova Z, Koska J, Huckova M, Ksinantova L, Imrich R, Vigas M, Trnovec T, Langer P, Sebokova E \& Klimes I. Insulin sensitivity indices: a proposal of cut-off points for simple identification of insulin-resistant subjects. Experimental and Clinical Endocrinology and Diabetes $2006114249-256$.

21 Hahn S, Tan S, Elsenbruch S, Quadbeck B, Herrmann BL, Mann K \& Janssen OE. Clinical and biochemical characterization of women with polycystic ovary syndrome in North Rhine-Westphalia. Hormone and Metabolic Research 200537 438-444.

22 Matthews DR, Hosker JP, Rudenski AS, Naylor BA, Treacher DF \& Turner RC. Homeostasis model assessment: insulin resistance and beta-cell function from fasting plasma glucose and insulin concentrations in man. Diabetologia 198528 412-419.

23 Katz A, Nambi SS, Mather K, Baron AD, Follmann DA, Sullivan G \& Quon MJ. Quantitative insulin sensitivity check index: a simple, accurate method for assessing insulin sensitivity in humans. Journal of Clinical Endocrinology and Metabolism 200085 2402-2410.

24 Matsuda M \& DeFronzo RA. Insulin sensitivity indices obtained from oral glucose tolerance testing: comparison with the euglycemic insulin clamp. Diabetes Care 199922 1462-1470.

25 Yilmaz M, Bukan N, Ersoy R, Karakoc A, Yetkin I, Ayvaz G, Cakir N \& Arslan M. Glucose intolerance, insulin resistance and cardiovascular risk factors in first degree relatives of women with polycystic ovary syndrome. Human Reproduction 200520 2414-2420.

26 Ascaso JF, Pardo S, Real JT, Lorente RI, Priego A \& Carmena R. Diagnosing insulin resistance by simple quantitative methods in subjects with normal glucose metabolism. Diabetes Care 200326 3320-3325.

27 Hrebicek J, Janout V, Malincikova J, Horakova D \& Cizek L. Detection of insulin resistance by simple quantitative insulin sensitivity check index QUICKI for epidemiological assessment and prevention. Journal of Clinical Endocrinology and Metabolism 2002 87 144-147.

28 Azziz R, Sanchez LA, Knochenhauer ES, Moran C, Lazenby J, Stephens KC, Taylor K \& Boots LR. Androgen excess in women: experience with over 1000 consecutive patients. Journal of Clinical Endocrinology and Metabolism 200489 453-462.

29 Souter I, Sanchez LA, Perez M, Bartolucci AA \& Azziz R. The prevalence of androgen excess among patients with minimal unwanted hair growth. American Journal of Obstetrics and Gynaecology $2004191759-767$.

30 Rosner W, Auchus RJ, Azziz R, Sluss PM \& Raff H. Position statement: Utility, limitations, and pitfalls in measuring testosterone: an Endocrine Society position statement. Journal of Clinical Endocrinology and Metabolism 200792 405-413.

31 Mathur RS, Moody LO, Landgrebbe S \& Williamson HO. Plasma androgens and sex hormone binding globulin in the evaluation of hirsute patients. Fertility and Sterility 198135 29-37.

32 Stumvoll M, Van Haeften T, Fritsche A \& Gerich J. Oral glucose tolerance test indexes for insulin sensitivity and secretion based on various availabilities of sampling times. Diabetes Care 200124 796-797.

33 Hanley AJ, Williams K, Gonzalez C, D'Agostino RB Jr, Wagenknecht LE, Stern MP \& Haffner SM. San Antonio Heart Study; Mexico City Diabetes Study; Insulin Resistance
Atherosclerosis Study. Prediction of type 2 diabetes using simple measures of insulin resistance: combined results from the San Antonio Heart Study, the Mexico City Diabetes Study, and the Insulin Resistance Atherosclerosis Study. Diabetes 200352 463-469 (erratum in Diabetes 200352 1306).

34 Radikova Z. Assessment of insulin sensitivity/resistance in epidemiological studies. Endocrine Regulations 200337 189-194.

35 Petra PH. The plasma sex steroid binding protein (SBP or SHBG). A critical review of recent developments on the structure, molecular biology and function. Journal of Steroid Biochemistry and Molecular Biology 199140 735-753.

36 Nestler JE, Powers LP, Matt DW, Steingold KA, Plymate SR, Rittmaster RS, Clore JN \& Blackard WG. A direct effect of hyperinsulinemia on serum sex hormone-binding globulin levels in obese women with the polycystic ovary syndrome. Journal of Clinical Endocrinology and Metabolism 1991 72 83-89.

37 Plymate SR, Jones RE, Matej LA \& Friedl KE. Regulation of sex hormone binding globulin (SHBG) production in Hep G2 cells by insulin. Steroids $1988 \mathbf{5 2} 339-340$.

38 Heald AH, Anderson SG, Ivison F, Riste L, Laing I, Cruickshank JK \& Gibson JM. Low sex hormone binding globulin is a potential marker for the metabolic syndrome in different ethnic groups. Experimental and Clinical Endocrinology and Diabetes 2005113 $522-528$.

39 Preziosi P, Barrett-Connor E, Papoz L, Roger M, Saint-Paul M, Nahoul K \& Simon D. Interrelation between plasma sex hormonebinding globulin and plasma insulin in healthy adult women: the telecom study. Journal of Clinical Endocrinology and Metabolism $199376283-287$.

40 Dahan MH \& Goldstein J. Serum sex hormone-binding globulin levels show too much variability to be used effectively as a screening marker for insulin resistance in women with polycystic ovary syndrome. Fertility and Sterility 200686 934-941.

41 Cousin P, Calemard-Michel L, Lejeune H, Raverot G, Yessaad N, Emptoz-Bonneton A, Morel Y \& Pugeat M. Influence of SHBG gene pentanucleotide TAAAA repeat and $\mathrm{D} 327 \mathrm{~N}$ polymorphism on serum sex hormone-binding globulin concentration in hirsute women. Journal of Clinical Endocrinology and Metabolism 200489 917-924.

42 Xita N, Tsatsoulis A, Chatzikyriakidou A \& Georgiou I. Association of the (TAAAA)n repeat polymorphism in the sex hormonebinding globulin $(S H B G)$ gene with polycystic ovary syndrome and relation to SHBG serum levels. Journal of Clinical Endocrinology and Metabolism $2003 \mathbf{8 8} 5976-5980$.

43 Bendlová B, Zavadilová J, Vanková M, Vejrazková D, Lukásová P, Vcelák J, Hill M, Cibula D, Vondra K, Stárka L \& Vrbíková J. Role of D $327 \mathrm{~N}$ sex hormone-binding globulin gene polymorphism in the pathogenesis of polycystic ovary syndrome. Journal of Steroid Biochemistry and Molecular Biology 2007104 68-74.

44 Ducluzeau PH, Cousin P, Malvoisin E, Bornet H, Vidal H, Laville M \& Pugeat M. Glucose-to-insulin ratio rather than sex hormone-binding globulin and adiponectin levels is the best predictor of insulin resistance in nonobese women with polycystic ovary syndrome. Journal of Clinical Endocrinology and Metabolism 200388 3626-3631.

45 Katsuki A, Sumida Y, Murashima S, Fujii M, Ito K, Tsuchihashi K, Murata K, Yano Y \& Shima T. Acute and chronic regulation of serum sex hormone-binding globulin levels by plasma insulin concentrations in male noninsulin-dependent diabetes mellitus patients. Journal of Clinical Endocrinology and Metabolism 199681 2515-2519.

46 Szurkowska M, Szafraniec K, Gilis-Januszewska A, Szybinski Z \& Huszno B. Insulin resistance indices in population-based study and their predictive value in defining metabolic syndrome. Przeglad Epidemiologiczny $2005 \mathbf{5 9} 743-751$.

47 Penesova A \& Radikova Z. Comparison of insulin sensitivity indices calculated from standard 3-sampled and frequently sampled oral glucose tolerance test. Endocrine Regulations 2004 38 167-171.

48 von Eckardstein A, Nofer JR \& Assmann G. High density lipoproteins and arteriosclerosis. Role of cholesterol efflux and reverse cholesterol transport. Arteriosclerosis, Thrombosis and Vascular Biology 200121 13-27. 
49 Pugeat M, Moulin P, Cousin P, Fimbel S, Nicolas MH, Crave JC \& Lejeune H. Interrelations between sex hormone-binding globulin (SHBG), plasma lipoproteins and cardiovascular risk. Journal of Steroid Biochemistry and Molecular Biology 199553 567-572.

50 Korytkowski MT, Krug EI, Daly MA, Deriso L, Wilson JW \& Winters SJ. Does androgen excess contribute to the cardiovascular risk profile in postmenopausal women with type 2 diabetes? Metabolism 200554 1626-1631.

51 Vermeulen A, Verdonck L \& Kaufmann JM. A Critical evaluation of simple methods for the estimation of free testosterone in serum. Journal of Clinical Endocrinology and Metabolism $1999 \mathbf{8 4}$ 3666-3672.

52 Mueller A, Cupisti S, Binder H, Hoffmann I, Beckmann MW \& Dittrich R. The role of albumin in the calculation of free and bioavailable testosterone in women with hyperandrogenemia. In Vivo 200620 403-407.

53 The Rotterdam ESHRE/ASRM-Sponsored PCOS consensus workshop group. Revised 2003 consensus on diagnostic criteria and long-term health risks related to polycystic ovary syndrome (PCOS). Human Reproduction $20041941-47$.
54 Mueller A, Dittrich R, Cupisti S, Beckmann MW \& Binder H. Is it necessary to measure free testosterone to assess hyperandrogenemia in women? The role of calculated free and bioavailable testosterone Experimental and Clinical Endocrinology and Diabetes 2006114 182-187.

55 Mueller A, Dittrich R, Binder H, Hoffmann I, Beckmann MW \& Cupisti S. Evaluation of recommended markers for assessing hyperandrogenemia in women classified as having polycystic ovary syndrome (PCOS) according to the revised 2003 diagnostic criteria. European Journal of Medical Research 2006 $11540-544$.

56 Cupisti S, Dittrich R, Binder H, Kajaia N, Hoffmann I, Maltaris T, Beckmann MW \& Mueller A. Influence of body mass index on calculated androgen parameters in adult women with hirsutism and PCOS. Experimental and Clinical Endocrinology and Diabetes $2007115298-302$.

Received 31 May 2007

Accepted 24 July 2007 